\title{
The coexpression and prognostic significance of c-MET, fibroblast growth factor receptor 2 , and human epidermal growth factor receptor 2 in resected gastric cancer: a retrospective study
}

\author{
This article was published in the following Dove Press journal: \\ OncoTargets and Therapy \\ 27 September 2016 \\ Number of times this article has been viewed
}

\author{
Yong-Xu Jia $1,2, *$ \\ Teng-Fei $\mathrm{Li}^{3, *}$ \\ Dan-Dan Zhang ${ }^{4}$ \\ Zong-Min Fan ${ }^{5}$ \\ Hui-Jie Fan ${ }^{1,2}$ \\ Jie Yan ${ }^{1,2}$ \\ Li-Juan Chen ${ }^{6}$ \\ Hong Tang ${ }^{6}$ \\ Yan-Ru Qin ${ }^{1,2}$ \\ Xing-Ya Li' \\ 'Department of Oncology, The First \\ Affiliated Hospital of Zhengzhou \\ University, ${ }^{2}$ Esophageal and Gastric \\ Cancer Center of Henan Province, \\ ${ }^{3}$ Department of Interventional \\ Radiology, ${ }^{4}$ Department of \\ Pathology, ${ }^{5}$ Henan Key Laboratory \\ for Esophageal Cancer Research, \\ The First Affiliated Hospital of \\ Zhengzhou University, ' Department \\ of Oncology, Tumor Hospital of Henan \\ Province, Zhengzhou, Henan, People's \\ Republic of China \\ *These authors contributed equally \\ to this work
}

Correspondence: Yan-Ru Qin; Xing-Ya Li Department of Oncology, The First Affiliated Hospital of Zhengzhou University, No I Jianshe Road, Zhengzhou, Henan, People's Republic of China

Email yanruqin@zzu.edu;

lixingya00I@I26.com

\begin{abstract}
Molecular-targeted therapy against tyrosine kinase receptors (RTKs) plays an important role in gastric cancer (GC) treatment. Understanding the correlation between RTK coexpression could better guide clinical drug use. In the present study, the coexpression status of c-MET, fibroblast growth factor receptor 2 (FGFR2), and human epidermal growth factor receptor 2 (HER2) in human GC and their clinical significance in clinical therapy were explored. Immunohistochemical (IHC) staining, quantitative real-time polymerase chain reaction and fluorescent in situ hybridization were performed in 143 cases of GC who had undergone gastrectomy without preoperative chemoradiotherapy. Their association with clinicopathological features and clinical prognosis was analyzed. The frequencies of c-MET, FGFR2, and HER2 overexpression were $47.6 \%$ (68/143), 34.3\% (49/143), and 10.5\% (15/143), respectively. In the RTK coexpression study, $30.1 \%$ of patients (43/143) were positive for only one RTK, $25.8 \%(37 / 143)$ were positive for two RTKs, 3.5\% (5/143) had triple-positive status, and $40.6 \%(58 / 143)$ had triple-negative status. In survival analysis, the overexpression of c-MET, FGFR2, and HER2 were significantly associated with overall survival (OS) $(P=0.018,0.004$, and 0.049 , respectively). In coexpression analysis, patients with triple-positive GC had the poorest OS $(P=0.013)$. In conclusion, RTK coexpression is significantly associated with poor clinical outcome in GC.
\end{abstract}

Keywords: c-MET, FGFR2, HER2, gastric cancer, coexpression, survival, target therapy

\section{Introduction}

Gastric cancer (GC) is the fourth most common cause of cancer death worldwide and the second most common cause of cancer-related death. ${ }^{1}$ Its incidence is estimated to be $\sim 930,000$ newly diagnosed cases each year with wide geographical variation, and $50 \%$ of new cases occur within Eastern Asia. ${ }^{2}$ Despite incidence and mortality declining globally following the substantial developments in early screening, surgery, and chemotherapy over the past two decades, GC remains an important health problem in the People's Republic of China. ${ }^{3}$ Radical gastrectomy is the only curative treatment for $\mathrm{GC}$, but recurrence is detected in $\sim 60 \%$ of patients after surgery. ${ }^{4}$ The long-term prognosis of GC remains dismal, with median overall survival (OS) of 8-10 months and a 5-year survival rate of $<20 \%$. 5,6

During the last decade, molecular-targeted therapies have made substantial progress against many common malignancies such as lung, colorectal, and breast cancer, but less progress against GC. In the Trastuzumab for Gastric Cancer (ToGA) study, ${ }^{7}$ 
a Phase III trial, trastuzumab improved the progress-free survival and OS of human epidermal growth factor receptor 2 (HER2)-positive advanced gastroesophageal junction cancer with statistical significance when combined with chemotherapy. Unfortunately, only $15 \%-20 \%$ of patients with GC is HER2-positive and can benefit clinically from anti-HER2-targeted therapy. ${ }^{8-10}$ HER2-negative patients, accounting for the biggest proportion of patients with GC, have no other option but conventional chemotherapy. Therefore, further studies are warranted to explore more targets and provide more clinical options for treating GC. Similar to HER2, tyrosine kinase receptors (RTKs) such as MET and fibroblast growth factor receptor 2 (FGFR2) play key roles in tumor proliferation, invasion, angiogenesis, and metastasis, and they are considered potential therapeutic targets or biomarkers of $\mathrm{GC} .{ }^{11-16}$

As a member of the RTK family, c-MET is encoded by the proto-oncogene $M E T$ and is expressed in epithelial and endothelial cells. ${ }^{17}$ By binding to the ligand of hepatocyte growth factor/scatter factor, c-MET triggers the activation of multiple steps in the signal transduction cascade involving the Ras/Raf/mitogen-activated protein kinase and phosphatidyl-inositol-3-kinase/AKT/mammalian target of rapamycin pathways, which typically regulate cell survival, proliferation, cytoskeleton, and mobility. ${ }^{18-23}$ Recently, several c-MET inhibitors such as monoclonal antibodies and small-molecule tyrosine kinase inhibitors have been investigated in clinical trials, and the initial results were optimistic. ${ }^{24}$ Therefore, therapies targeting the c-MET signaling pathway are becoming a promising treatment strategy for GC, and they provide a novel method for overcoming resistance to HER2-targeting agents.

The FGFR family also belongs to the RTK family and is divided into four subtypes: FGFR1, 2, 3, and 4. Dysregulation of FGFR subtype signaling has been observed in malignancies with variants including gene mutation, gene amplification, and protein overexpression. ${ }^{25}$ FGFR1 gene amplification has been reported in ovarian cancer, breast cancer, bladder cancer, oral squamous carcinoma, and rhabdomyosarcoma. ${ }^{14,26}$ FGFR2 gene mutations have been observed in endometrial cancer, and FGFR2 gene amplification and protein overexpression have been detected in GC. ${ }^{27-30}$ FGFR3 gene mutation and amplification have been detected in bladder cancer, and FGFR3 translocation has been detected in myeloma. ${ }^{14,31}$ Preclinical studies have demonstrated that several monoclonal antibodies and small-molecule inhibitors of FGFR are effective antitumor drugs. ${ }^{32-35}$ Together, these data suggest that the FGFR signaling pathway is correlated with tumor growth, invasion, metastasis, and poor prognosis and may be a potential target in cancer treatment.

However, most previous studies have focused on the protein overexpression or gene amplification profile of single biomarkers in one cohort. ${ }^{36-38}$ The relation between the pathological features, clinical factors, and prognosis of these promising RTKs has rarely been evaluated simultaneously in Chinese patients. In this study, the coexpression of three RTKs (c-MET, FGFR2, and HER2) and their correlation with clinical prognosis in 143 cases of GC patients were retrospectively investigated.

\section{Materials and methods \\ Ethics statement}

Written informed consent was obtained from each patient for the publication of this paper and any accompanying images. This study was approved by the ethics committee of Zhengzhou University. The procedures followed were in accordance with the Helsinki Declaration of 1975, as revised in 1983.

\section{Patients and tumor specimens}

A total of 143 patients with GC with complete medical records from the First Affiliated Hospital of Zhengzhou University from 2011 to 2012 were enrolled. All of them had received total or subtotal gastrectomy with lymphadenectomy, with pathologically confirmed diagnosis of primary gastric or gastroesophageal junction adenocarcinoma and no history of neoadjuvant therapy. All specimens were routinely fixed in buffered formalin, embedded in paraffin blocks, and sectioned into continuous $4-\mu \mathrm{m}$ tissue sections for immunohistochemical (IHC) examination. Of the 143 patients, 110 patients were male and 33 were female, with the age ranging from 42 to 86 years; the average age was $60 \pm 9.55$ years and the median age was 62 years. Seventy-two patients $(50.3 \%)$ died during the follow-up period. Tumor classification was according to the Lauren classification; ${ }^{39}$ tumor-nodes-metastasis staging was according to the International Union against Cancer staging system. ${ }^{40}$ Patient information and tumor characteristics including age; sex; tumor location, type, differentiation; depth of invasion; lymph node metastasis; and clinical stage are summarized in Table 1.

\section{Immunohistochemistry}

IHC analysis was performed on the $4-\mu \mathrm{m}$ tissue sections. The procedure was in accordance with the IHC streptavidin/ peroxidase detection kit instructions. The sections were deparaffinized in xylene and dehydrated with graded ethanol, then microwaved in citric buffer ( $\mathrm{pH}$ 6.0) for antigen retrieval for 15 minutes. After naturally cooled, endogenous peroxidase 
Table I Correlation between clinicopathological features and three RTKs in GC

\begin{tabular}{|c|c|c|c|c|c|c|c|c|c|c|}
\hline \multirow{2}{*}{$\begin{array}{l}\text { Clinicopathological } \\
\text { features }\end{array}$} & \multirow{2}{*}{$\begin{array}{l}\text { Number } \\
\text { of cases }\end{array}$} & \multicolumn{2}{|c|}{ c-MET } & \multirow[t]{2}{*}{$P$-value } & \multicolumn{2}{|c|}{ FGFR2 } & \multirow[t]{2}{*}{$P$-value } & \multicolumn{2}{|c|}{ HER2 } & \multirow[t]{2}{*}{$P$-value } \\
\hline & & $(+)$ & $(-)$ & & $(+)$ & $(-)$ & & $(+)$ & $(-)$ & \\
\hline \multicolumn{11}{|l|}{ Age (years) } \\
\hline$>60$ & 81 & 39 & 42 & & 27 & 54 & & 10 & 71 & \\
\hline$<60$ & 62 & 29 & 33 & 0.870 & 22 & 40 & 0.788 & 5 & 57 & 0.408 \\
\hline \multicolumn{11}{|l|}{ Sex } \\
\hline Male & 110 & 52 & 58 & & 37 & 73 & & 13 & 97 & \\
\hline Female & 33 & 16 & 17 & 0.903 & 12 & 21 & 0.772 & 2 & 31 & 0.344 \\
\hline \multicolumn{11}{|l|}{ Tumor location } \\
\hline Cardia & 27 & 13 & 14 & & 7 & 20 & & 0 & 27 & \\
\hline Body & 106 & 52 & 54 & & 38 & 68 & & 14 & 92 & \\
\hline Antrum & 10 & 3 & 7 & 0.513 & 4 & 6 & 0.578 & I & 9 & 0.135 \\
\hline \multicolumn{11}{|l|}{ Tumor differentiation } \\
\hline Well & 45 & 9 & 36 & & 6 & 39 & & 5 & 40 & \\
\hline Modern & 33 & 14 & 19 & & 9 & 24 & & 6 & 27 & \\
\hline Poor & 65 & 45 & 20 & $<\mathbf{0 . 0 0 1}$ & 33 & 32 & $<0.00 \mathrm{I}$ & 4 & 61 & 0.183 \\
\hline \multicolumn{11}{|l|}{ Tumor type } \\
\hline Intestinal & 80 & 22 & 58 & & 17 & 63 & & 10 & 70 & \\
\hline Diffuse & 56 & 41 & 15 & & 28 & 28 & & 4 & 52 & \\
\hline Mixed & 7 & 5 & 2 & $<0.001$ & 4 & 3 & 0.001 & I & 6 & 0.069 \\
\hline \multicolumn{11}{|l|}{ Depth of invasion } \\
\hline $\mathrm{pTI}$ & 23 & 3 & 20 & & 3 & 20 & & 0 & 23 & \\
\hline pT2 & 26 & 10 & 16 & & 7 & 19 & & 2 & 24 & \\
\hline $\mathrm{pT} 3$ & 92 & 54 & 38 & & 38 & 54 & & 13 & 79 & \\
\hline $\mathrm{pT} 4$ & 2 & 1 & 1 & 0.001 & 1 & 1 & 0.058 & 0 & 2 & 0.045 \\
\hline \multicolumn{11}{|l|}{$\mathrm{LN}$ metastasis } \\
\hline No & 94 & 29 & 65 & & 20 & 74 & & 6 & 88 & \\
\hline Yes & 49 & 39 & 10 & $<0.001$ & 29 & 20 & $<0.001$ & 9 & 40 & 0.026 \\
\hline \multicolumn{11}{|l|}{ TNM stage } \\
\hline I & $4 I$ & 11 & 30 & & 6 & 35 & & 1 & 40 & \\
\hline II & 82 & 38 & 44 & & 31 & 51 & & 9 & 73 & \\
\hline III & 20 & 19 & 1 & $<0.001$ & 12 & 8 & 0.001 & 5 & 15 & 0.026 \\
\hline Total & 143 & 68 & 75 & & 49 & 94 & & 15 & 121 & \\
\hline
\end{tabular}

Note: $P<0.05$ are shown in bold.

Abbreviations: FGFR2, fibroblast growth factor receptor 2; HER2, human epidermal growth factor receptor 2; LN, lymphnode; TNM, tumor, lymphnode, metastasis.

was eliminated with 3\% peroxide-methanol at room temperature for 5 minutes. Nonspecific staining was avoided with blocking buffer (normal goat serum) at room temperature for 20 minutes. The sections were incubated with diluted primary antibody for 2 hours at $37^{\circ} \mathrm{C}$. The final dilution of the rabbit antihuman FGFR2 antibody (ab10648; Abcam) was 1:500, mouse antihuman HER2 antibody (ab79205; Abcam) was 1:500, and that of the rabbit antihuman c-MET antibody (ab39075; Abcam) was 1:200. Goat antirabbit immunoglobulin G (Beijing Zhongshan Golden Bridge Biotechnology Co., Ltd. Beijing, People's Republic of China) was added and incubated for another 30 minutes at $37^{\circ} \mathrm{C}$. Afterward, the sections were incubated with streptavidin-horseradish peroxidase (Beijing Zhongshan Golden Bridge Biotechnology Co., Ltd.) at $37^{\circ} \mathrm{C}$ for 30 minutes before being stained with 3,3-diaminobenzidine (Beijing Zhongshan Golden Bridge Biotechnology Co., Ltd.) for 10 minutes. Finally, hematoxylin was used for counterstaining, followed by dehydration, clearing, and mounting with neutral gum. A gentle rinse with washing buffer was conducted during the experiment if necessary. All steps were carried out in a moist chamber. Known c-MET-positive and FGFR2-positive lung adenocarcinoma specimens were used as the respective positive controls. In the negative control group, the antibody was replaced with phosphate-buffered saline.

\section{Evaluation of IHC staining}

Each section was assessed by semiquantitative analysis. Consensus and recommendations according to Hofmann's criteria were used in HER2 scoring for $\mathrm{GC}^{8}$ : 0 : no reactivity or membranous reactivity in $<10 \%$ of cells; $1+$ : faint/barely perceptible membranous reactivity in $>10 \%$ of cells; cells are reactive only in part of their membrane; $2+$ : weak to moderate complete or basolateral membranous reactivity in $>10 \%$ of tumor cells; $3+$ : moderate to strong complete or basolateral membranous reactivity in $>10 \%$ of tumor cells. 
C-MET and FGFR2 were assessed by semiquantitative analysis according to clinical trial criteria. ${ }^{41,42}$ The intensity of staining was scored as 0 (no staining), 1 (weak staining), 2 (moderate staining), or 3 (strong staining). The proportion of positive cells was scored as $0(0 \%$ positive), $1(<10 \%$ positive), 2 ( $10 \%-50 \%$ positive), or 3 ( $>50 \%$ positive). A total score was derived by adding the proportional score; an intensity score $\geq 3$ was regarded as high expression; otherwise, it was defined as low expression. All slides were independently evaluated and scored by two pathologists blinded to the patient data. Cases with disputed scores were reexamined under a discussion microscope.

\section{Quantitative real-time polymerase chain reaction}

Total RNA from tumor tissues was extracted using Trizol (Thermo Fisher Scientific, Waltham, MA, USA) according to the manufacturer's instructions. Real-time quantitative polymerase chain reaction (qPCR) was carried out to detect levels of HER2, c-MET, and FGFR2 using a SYBR Green PCR Kit and an ABI 7900HT Fast Real-Time PCR System (Thermo Fisher Scientific). Glyceraldehyde-3-phosphate dehydrogenase (GAPDH) was used as internal control. The relative expression level (defined as fold change) of target gene $\left(2^{\Delta \Delta C t}\right)$ was normalized to the endogenous GAPDH reference and related to the amount of target gene in control sample, which was defined as the calibrator at 1.0. The assays were done in triplicate.

\section{Dual-color silver in situ hybridization}

HER2 silver in situ hybridization was performed using an automatic instrument (BenchMark XT; Ventana Medical Systems, Tucson, AZ, USA) according to the manufacturer's protocol. Testing for the HER2 gene and CEP17 was performed on the sections in each case. $H E R 2$ gene amplification was defined as a $H E R 2 / C E P 17$ ratio $\geq 2$.

\section{Statistics}

Statistical analysis was performed using IBM SPSS 20.0 statistical software (IBM Corporation, Armonk, NY, USA); count data were analyzed using the $\chi^{2}$ test. All tests were two sided. The OS was calculated from the date of surgery to death for any reason. The survival curves were determined by Kaplan-Meier analysis, and log-rank tests were used to compare the differences among curves. Univariate and multivariate Cox proportional hazards regression model analysis was used to evaluate the parameters associated with the hazard ratio (HR). $P<0.05$ was considered statistically significant.

\section{Results \\ Correlation between clinicopathological features and three RTKs in GC}

Among the three RTKs, the expressions of both c-MET and FGFR2 were significantly correlated with tumor type $(P<0.001$ and 0.001 , respectively), tumor differentiation $(P<0.001,<0.001$, respectively), depth of invasion ( $P=0.001,0.058$, respectively), lymph node metastasis $(P<0.001,<0.001$, respectively), and clinical stage $(P<0.001,0.001$, respectively). However, they were not significantly associated with age, sex, and tumor location $(P>0.05)$. HER 2 overexpression was significantly related with depth of invasion $(P=0.045)$, lymph node metastasis $(P=0.026)$, and clinical stage $(P=0.026)$ but was not significantly associated with age, sex, tumor location, tumor type, and tumor differentiation $(P>0.05)$. The clinicopathological data for the three RTKs are shown in Table 1.

\section{HER2, c-MET, and FGFR2 overexpression in GC}

HER2, c-MET, and FGFR2 overexpression in the 143 cases is determined using IHC. HER2 staining was mainly in the cell membrane, but c-MET and FGFR2 staining was mainly in the cytoplasm and nucleus. Figure 1 depicts representative photomicrographs of the IHC analysis. Among the 143 cases, 22 cases (15\%) were detected with HER2 2+ or 3+: $109(76 \%)$ scored 0, 12 (8\%) scored 1+, 14 (10\%) scored 2+, and $8(6 \%)$ scored 3+. Only 15 cases (10.5\%) were with HER2 IHC $3+$ or IHC $2+$, but fluorescent in situ hybridization amplification were considered to be HER2 positive (Figure 2). C-MET positivity ( $\mathrm{IHC} 2+$ or $3+$ ) was detected in 68 cases $(48 \%)$ : 53 (37\%) scored 0, 22 (15\%) scored 1+, $45(29 \%)$ scored 2+, and $23(16 \%)$ scored $3+$. FGFR2 positivity (IHC2+ or $3+$ ) was detected in 49 cases (34\%): 76 (60\%) scored 0, 18 (13\%) scored 1+, 35 (24\%) scored 2+, and 14 (10\%) scored 3+. The HER2, c-MET, and FGFR2 overexpression is listed in detail in Table 2, and the representative immunostaining is depicted in Figure 1. The mRNA expression of HER2, c-MET, and FGFR2 was initially tested in 42 pairs of GC tumors and their corresponding nontumor tissues by qPCR. Upregulation of HER2, c-MET, and FGFR2 was detected in 18 of $42(42.85 \%), 15$ of $42(35.17 \%)$, and 16 of $42(38.09 \%)$ of GC tumors compared with their normal counterparts $(P<0.0001$; Figure 3$)$.

\section{Characteristics of HER2, c-MET, and FGFR2 coexpression}

In the coexpression study of the three RTKs, 43 patients (30.1\%) were positive for only one RTK, 37 patients (25.8\%) 

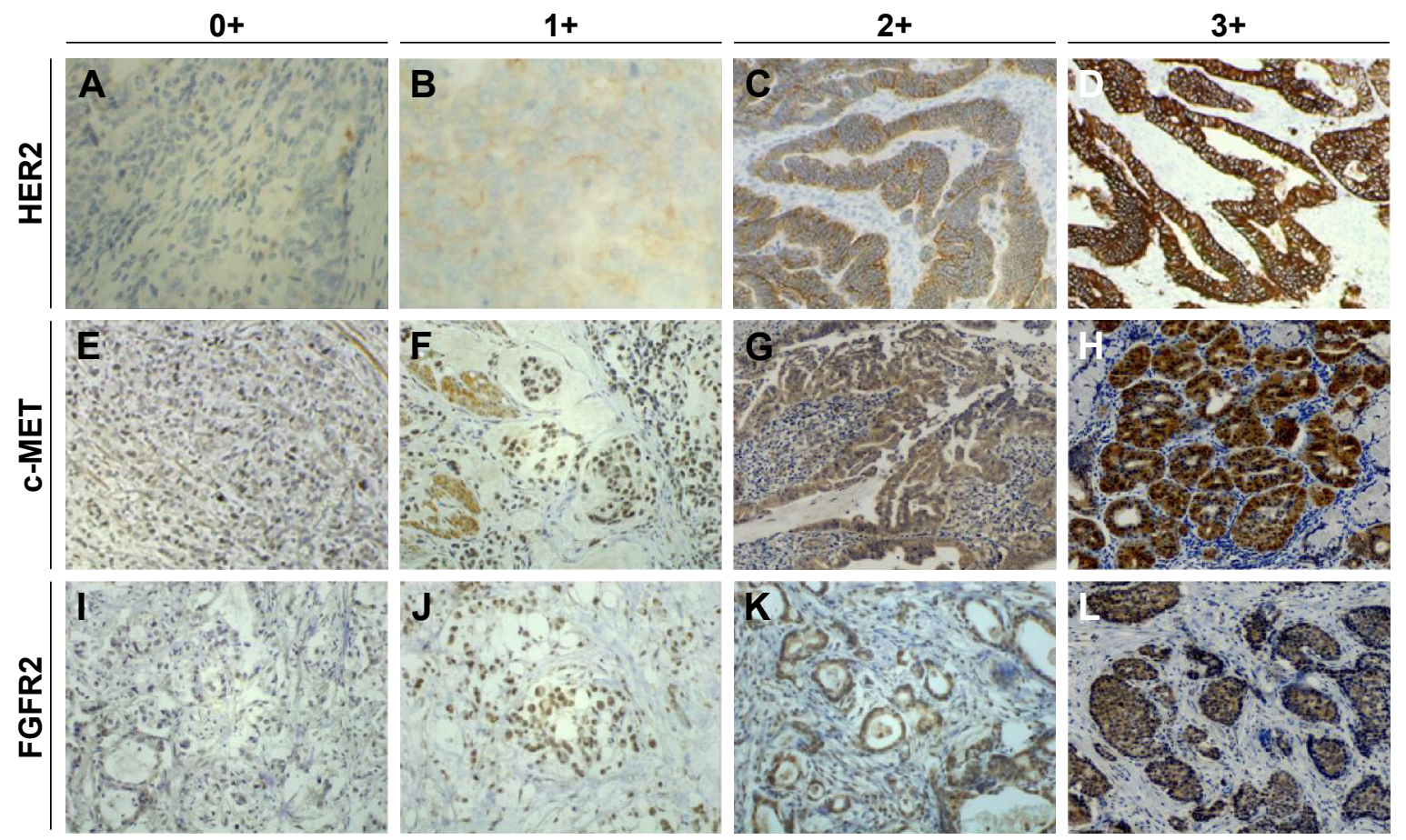

Figure I Evaluation of immunohistochemical staining for c-MET, FGFR2, and HER2 $(\times 400)$.

Notes: Immunostaining scores of $0+, \mathrm{I}+, 2+$, and $3+$ indicating overexpression of HER2 (A-D), c-MET (E-H), and FGFR2 (I-L), respectively.

Abbreviations: FGFR2, fibroblast growth factor receptor 2; HER2, human epidermal growth factor receptor 2.

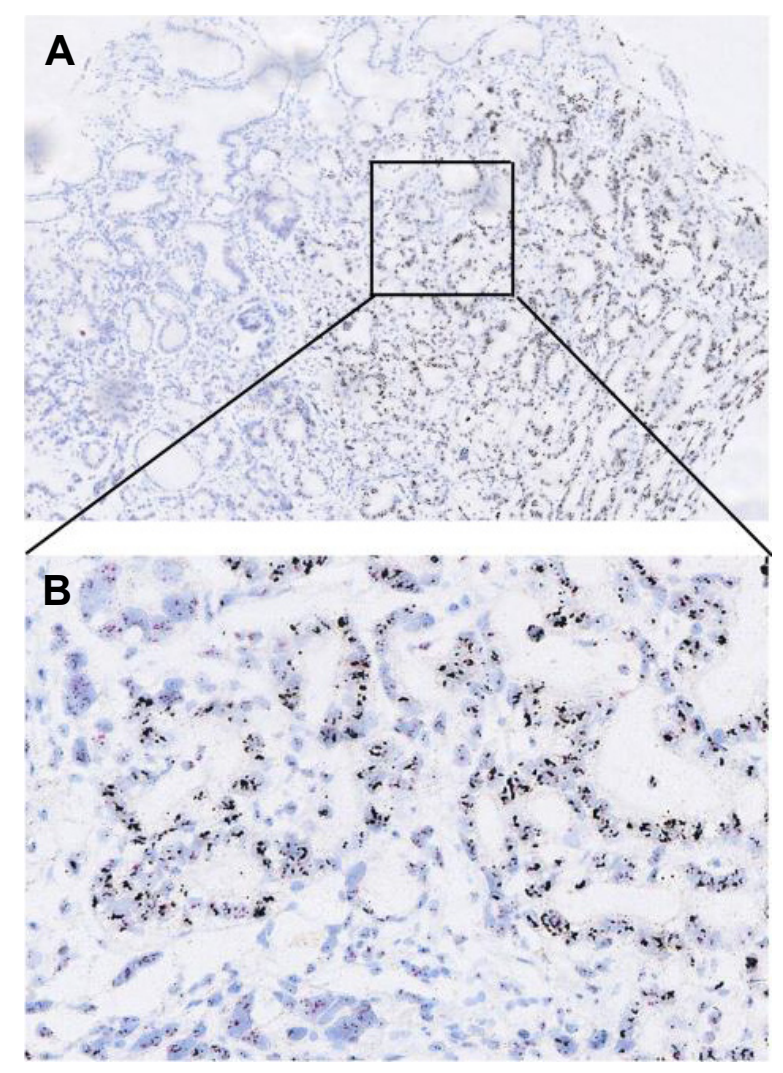

Figure 2 Representative features of SISH of HER2 GCN gain. Notes: (A) HER2 GCN gain $(\times 200)$, (B) HER2 GCN gain $(\times 400)$.

Abbreviations: GCN, gene copy number; HER2, human epidermal growth factor receptor 2; SISH, silver in situ hybridization. were simultaneously positive for two RTKs, five patients (3.5\%) had triple-positive status, and 58 patients (40.6\%) had triple-negative status. Regarding the coexpression of two RTKs specifically, 34 patients $(23.8 \%)$ were c-MET+/ FGFR2+, ten patients (7\%) were c-MET+/HER2+, and eight patients $(5.6 \%)$ were FGFR2+/HER2+. The details of the coexpression are depicted in Figures 4 and 5.

\section{Clinical outcomes of three RTKs}

We performed survival analyses in the 143 patients. The median follow-up time was $42 \pm 13.7$ months, and there were 72 deaths. The Kaplan-Meier curves for OS according to each RTK are shown in Figure 5. The OS between c-MET-positive and c-MET-negative patients was significantly different (median, 27 months [95\% confidence interval $\{\mathrm{CI}\}, 24.4-30.9$ months] vs 32 months [95\% CI, 29.4-35.4 months]; HR, 1.14, log-rank $P=0.018$; Figure $6 \mathrm{~A}$ ); for FGFR2, there was also a significant difference in OS between FGFR2-positive and FGFR2-negative patients (median, 24 months [95\% CI, 19.1-30.3 months] vs 31 months [ $95 \%$ CI, 28.7-33.5 months]; HR, 1.14; log-rank $P=0.012$; Figure 6B). Similar to c-MET and FGFR2, there was a significant difference between OS in HER2-positive and HER2-negative patients (median, 23 months [95\% CI, 17.8-30.3 months] vs 31 months [ $95 \%$ CI, 28.7-33.3 months]; HR, 1.14; log-rank $P=0.049$; Figure 6C). 
Table 2 IHC scores of HER2, c-MET, and FGFR2

\begin{tabular}{|c|c|c|c|c|c|c|c|c|}
\hline \multirow{2}{*}{$\begin{array}{l}\text { IHC } \\
\text { score }\end{array}$} & \multicolumn{2}{|l|}{$\mathbf{0}_{+}$} & \multicolumn{2}{|l|}{ I+ } & \multicolumn{2}{|l|}{$2+$} & \multicolumn{2}{|l|}{$3+$} \\
\hline & No & Percentage & No & Percentage & No & Percentage & No & Percentage \\
\hline HER2 & 109 & 76 & 12 & 8 & 14 & 10 & 8 & 6 \\
\hline c-MET & 53 & 37 & 22 & 15 & 45 & 31 & 23 & 16 \\
\hline FGFR2 & 76 & 32 & 18 & 12 & 35 & 24 & 14 & 10 \\
\hline
\end{tabular}

Abbreviations: FGFR2, fibroblast growth factor receptor 2; HER2, human epidermal growth factor receptor 2; IHC, immunohistochemical.

\section{Coexpression and survival}

In the subclassification based on all possible combinations, patients who were both c-MET- and FGFR2-positive had significantly poorer outcomes than the other patients (median, 26 months [95\% CI, 21.7-31.0 months] vs 31 months [95\% CI, 28.8-33.8 months]; HR, 1.14; log-rank $P=0.036$; Figure 6D). Patients who were both c-MET- and HER2positive had significantly worse survival as compared to the other patients (median, 19 months [95\% CI, 11.7-23.2 months] vs 31 months [95\% CI, 28.7-33.3 months]; log-rank $P=0.010$; Figure 6E). Patients who were both FGFR2- and HER2-positive had worse prognosis compared with the other patients (median, 21 months [95\% CI, 11.4-29.6 months] vs 31 months [95\% CI, 28.5-33.1 months]; log-rank $P=0.010$; Figure 6F). Similarly, patients with triple-positive tumors had significantly poorer clinical results than the other patients did (median, 21 months [95\% CI, 11.1-31.5 months] vs 31 months [95\% CI, 28.3-32.9 months]; log-rank $P=0.033$; Figure 6G). In the subgroup analysis, triple-positive patients had the poorest OS, and triple-negative patients had the best survival as compared to patients with overexpression of one or two RTKs ( $P=0.013$; Figure $6 \mathrm{H})$.

\section{Cox multivariate regression analysis}

Univariate analysis determined that tumor type, location, differentiation; depth of invasion; lymph node metastasis; clinical stage; and c-MET, FGFR2, and HER2 overexpression had significant prognostic value $(P<0.05)$. However, the differences disappeared in multivariate analysis (Table 3 ).

\section{Discussion}

Previous studies have reported that the frequency of IHC detection of HER2 overexpression in GC varies from $10 \%$ to $22.1 \%,{ }^{43,44}$ that of c-MET varies from $9 \%$ to $83 \%,{ }^{45,46}$ and that of FGFR 2 varies from $10 \%$ to $61 \% .{ }^{47,48}$ The varied range of overexpression of these three RTKs is likely due to differing methods, lack of standardized diagnosis criteria, tumor sample heterogeneity, and possible geographic differences. Considering these factors, whole tissue sections instead of tissue microarrays were examined, using surgical specimens instead of biopsy specimens in the present study to reduce the false-negative rate caused by tumor heterogeneity. In addition, new diagnostic criteria and antibodies that are employed in current clinical trials to improve IHC accuracy were used. In the present study, IHC analysis was performed in a large Chinese cohort, and HER2, c-MET, and FGFR2 overexpression in $10.5 \%(15 / 143), 47.6 \%$ (68/143), and $34.3 \%$ (49/143) of cases, respectively, was found, which is within the range of previous reports. Meanwhile, our results confirm the findings that Chinese patients with GC have a lower HER2-positive rate $(10 \%-15 \%)$ than White patients do $(22.1 \%))^{49,50}$

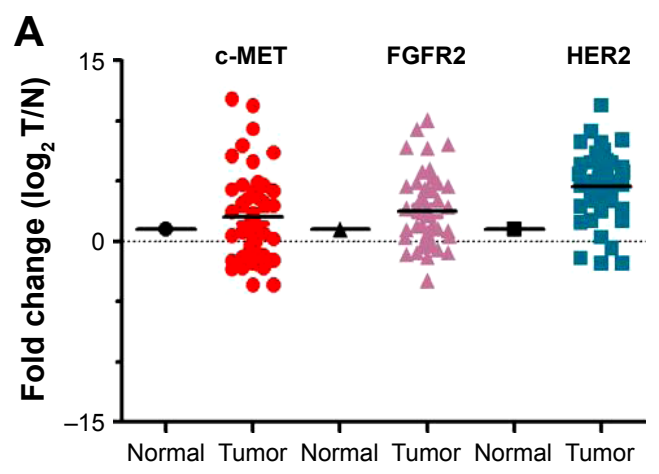

B

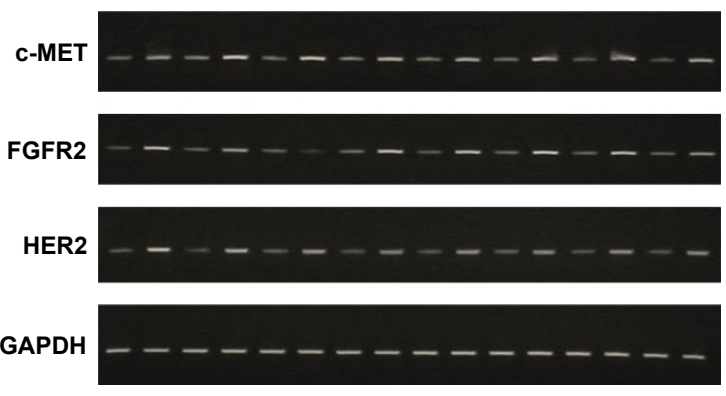

Figure 3 Upregulation of mRNA level of HER2, c-MET, and FGFR2 in 42 cases of GC.

Notes: (A) Relative expression of HER2, c-MET, and FGFR2 in GC cancer. Expression of HER2, c-MET, and FGFR2 in 42 primary GC cases was compared by qRT-PCR between tumor tissues and their paired nontumorous tissues. GAPDH was set as internal control. (B) The expression of HER2, c-MET, and FGFR2 in eight gastric cancer tissues was compared with the corresponding nontumorous tissues (normal) by RT-PCR.

Abbreviations: FGFR2, fibroblast growth factor receptor 2; GAPDH, glyceraldehyde-3-phosphate dehydrogenase; GC, gastric cancer; HER2, human epidermal growth factor receptor 2; qRT-PCR, quantitative real-time polymerase chain reaction. 


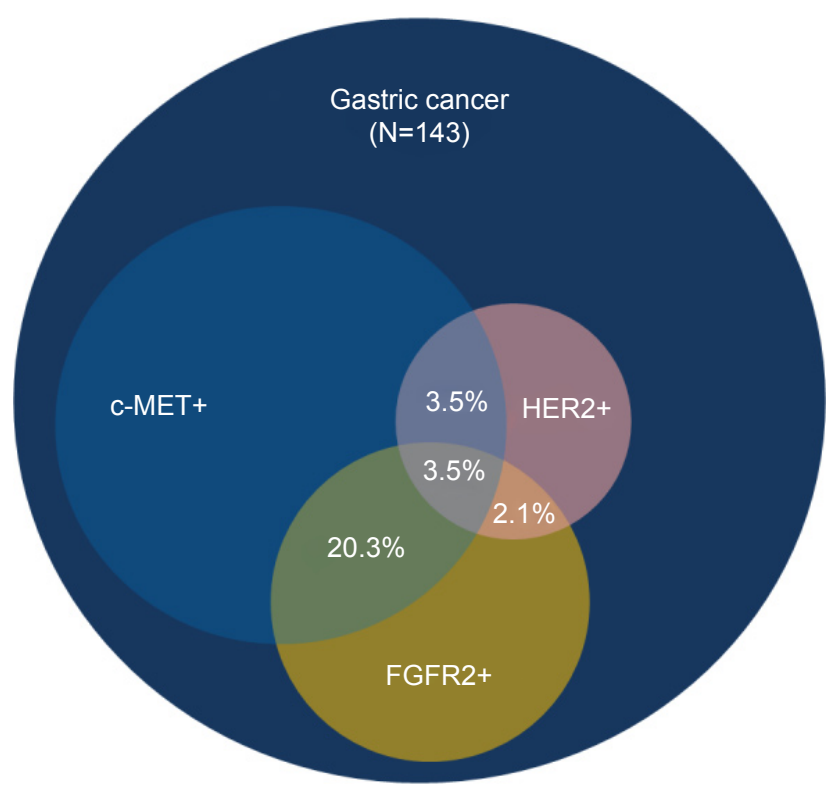

Figure 4 The relationship among three RTKs in patients with GC.

Note: The percentages of coexpression among HER2, FGFR2, and c-MET in patients with GC are depicted.

Abbreviations: FGFR2, fibroblast growth factor receptor 2; GC, gastric cancer HER2, human epidermal growth factor receptor 2; RTK, tyrosine kinase receptor.
Based on the relationship between the clinicopathological features and the overexpression of the three RTKs, it was observed that both c-MET and FGFR2 overexpression was more frequently related with diffuse tumor subtype, poor tumor differentiation, depth of tumor invasion, lymph node metastasis, and clinical stage, which is consistent with the findings of previous studies. ${ }^{51-53}$ Moreover, HER2 overexpression and well-differentiated tumors were significantly correlated. However, HER2 overexpression was not associated with tumor subtype, depth of tumor invasion, lymph node metastasis, and clinical stage. Despite previous research demonstrating that HER 2 is more frequently overexpressed in the gastroesophageal junction than in the stomach, ${ }^{10}$ there was no significant difference between HER2 overexpression and the location of GC in our study. Different populations, antibodies, and diagnostic criteria maybe the leading causes of the difference, especially as our cohort contained a large proportion of patients with diffuse GC with low HER2 expression. Our findings are supported by several studies that evaluated HER2 by performing IHC as well as gene amplification. ${ }^{54-56}$
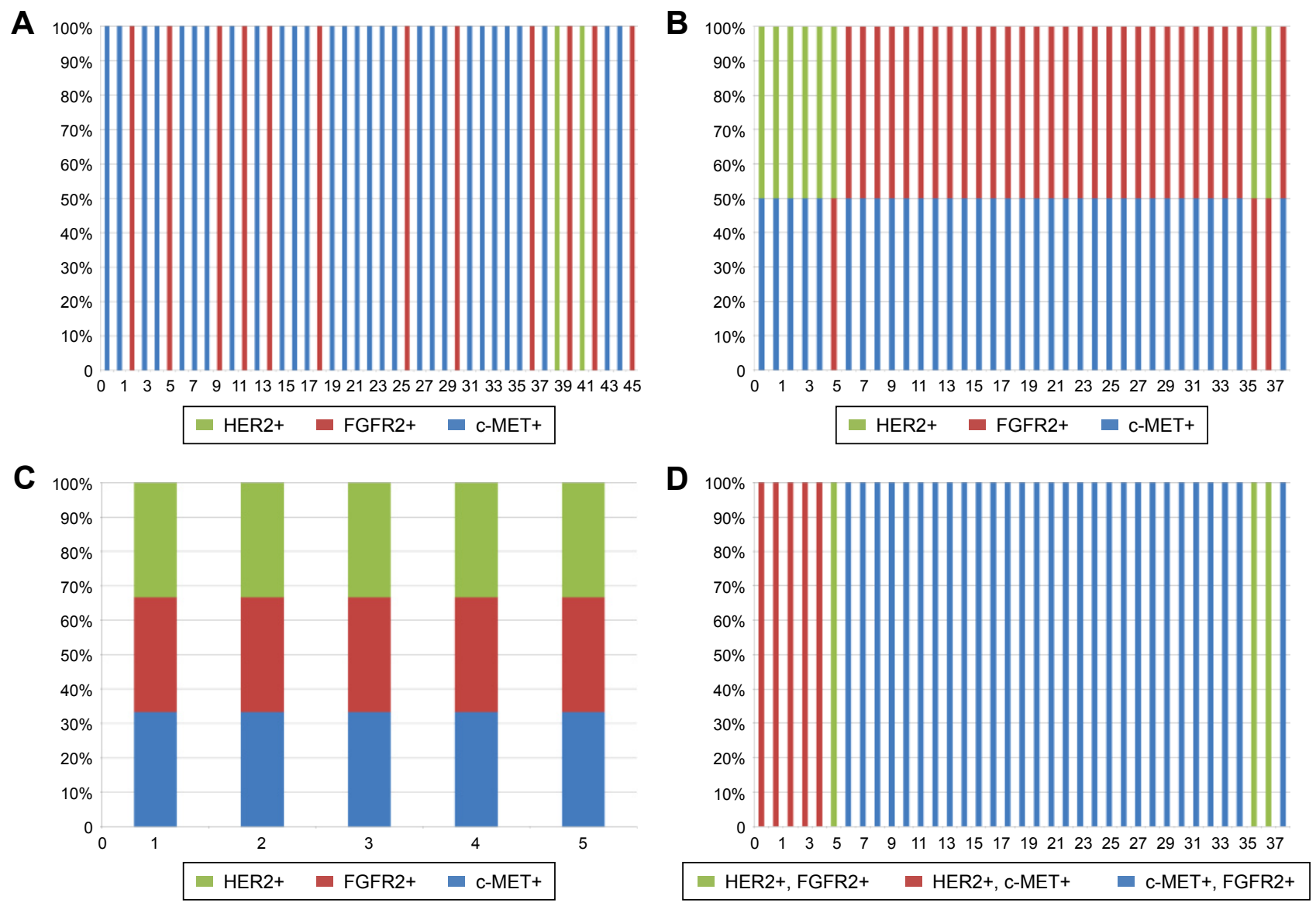

Figure 5 Coexpression status of RTKs.

Notes: Expression of c-MET, FGFR2, and HER2 in one RTK-positive (A) and two RTK-positive (B) patients, respectively. Coexpression of the three RTKs in triple-positive (C) and two RTK-positive (D) patients.

Abbreviations: FGFR2, fibroblast growth factor receptor 2; HER2, human epidermal growth factor receptor 2; RTK, tyrosine kinase receptor. 
A
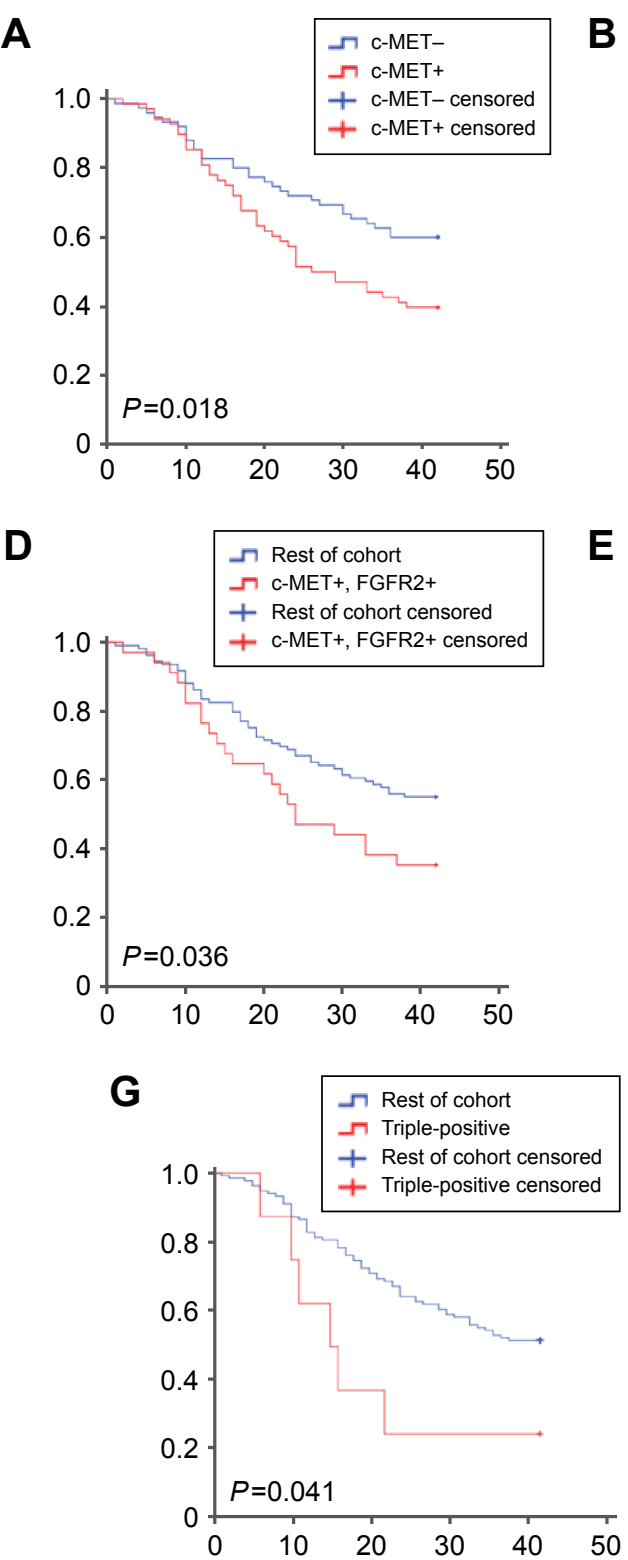

B

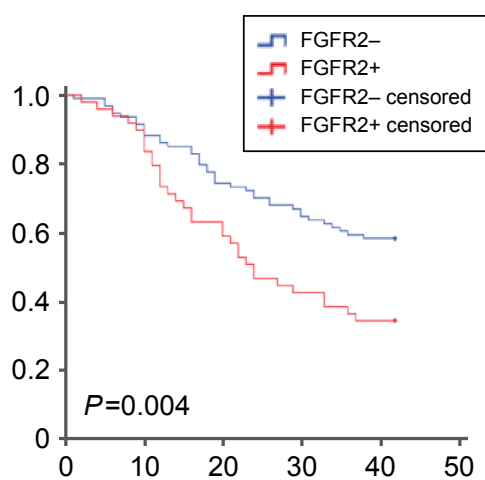

E

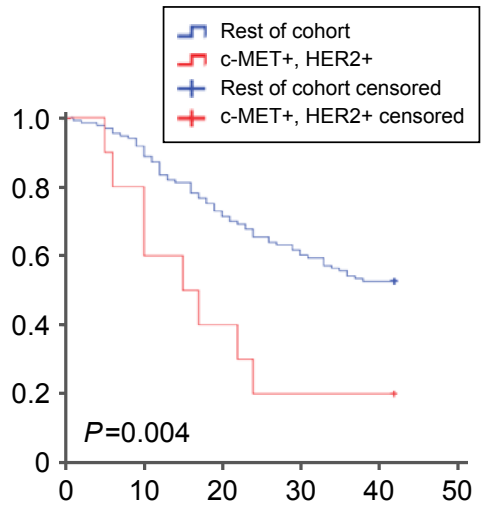

H

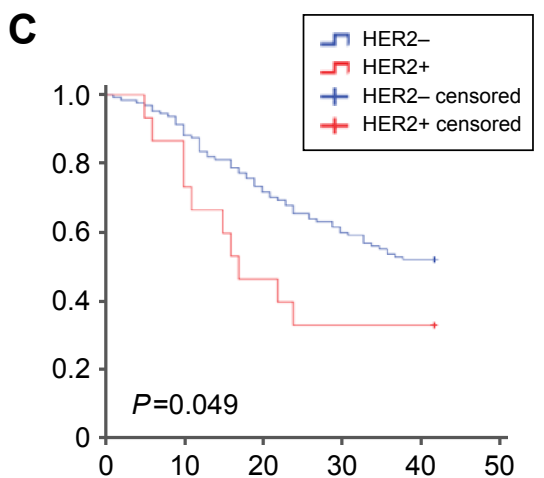

F

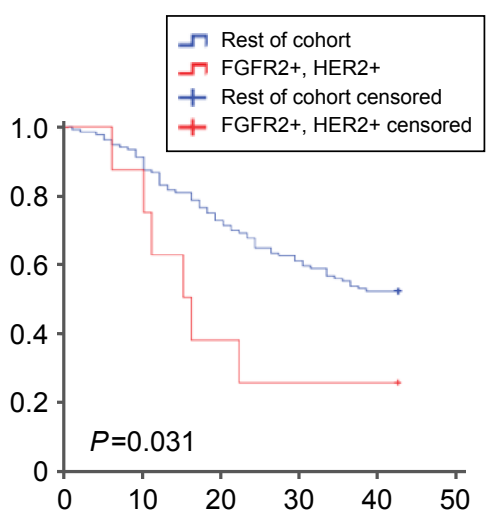

Figure 6 OS curves stratified by RTK coexpression.

Notes: OS analysis according to c-MET (A), FGFR2 (B), and HER2 (C) status, c-MET+/FGFR2+ (D), c-MET+/HER2+ (E), FGFR2+/HER2+ (F), and triple-positive status (G), and number of RTKs $(\mathbf{H})$.

Abbreviations: FGFR2, fibroblast growth factor receptor 2; HER2, human epidermal growth factor receptor 2; OS, overall survival; RTK, tyrosine kinase receptor.

Table 3 Cox proportional hazard regression analyses for OS

\begin{tabular}{|c|c|c|c|c|}
\hline \multirow[t]{2}{*}{ Clinical features } & \multicolumn{2}{|l|}{ Univariate analysis } & \multicolumn{2}{|c|}{ Multivariate analysis } \\
\hline & HR $(95 \% \mathrm{Cl})$ & $P$-value & HR $(95 \% \mathrm{Cl})$ & $P$-value \\
\hline Tumor location & $0.618(0.392-0.972)$ & 0.037 & $0.554(0.346-0.887)$ & 0.054 \\
\hline Tumor differentiation & $0.654(0.49 \mid-0.869)$ & 0.003 & $0.654(0.416-1.027)$ & 0.065 \\
\hline Tumor type & $1.590(1.093-2.313)$ & 0.015 & $1.123(0.654-1.955)$ & 0.682 \\
\hline Tumor invasion & $2.070(1.403-3.053)$ & 0.000 & $1.939(1.228-3.061)$ & 0.004 \\
\hline LN metastasis & $1.935(1.210-3.094)$ & 0.006 & $\mathrm{I} .565(0.88 \mathrm{I}-2.779)$ & 0.026 \\
\hline c-MET positive & $1.920(1.195-3.086)$ & 0.007 & $0.834(0.456-1.542)$ & 0.554 \\
\hline FGFR2 positive & $1.945(1.217-3.109)$ & 0.005 & $0.822(0.464-1.457)$ & 0.502 \\
\hline HER2 positive & $1.926(0.985-3.766)$ & 0.045 & $2.896(1.392-6.024)$ & 0.004 \\
\hline
\end{tabular}

Note: $P<0.05$ are shown in bold.

Abbreviations: Cl, confidence interval; FGFR2, fibroblast growth factor receptor 2; HER2, human epidermal growth factor receptor 2; HR, hazard ratio; LN, lymphnode. 
Regarding the RTK protein expression patterns as determined by IHC, it was found that staining location was correlated with tumor differentiation. In the present study, c-MET and FGFR2 overexpression was mainly found in poorly differentiated tumors and with cytoplasmic and nuclear staining. On the contrary, HER2 was always found in well-differentiated tumors and with membrane staining. Although the exact reason for the discrepancy is unknown, our results are confirmed by recent studies. Wiedlocha et al reported evidence that FGFR2 translocation from the cytoplasm to the nucleus is imperative for inducing cell proliferation in in vitro studies. ${ }^{57}$ In addition, Behrens et al indicated that cytoplasmic overexpression of FGFR2 is related to better clinical outcome but that nuclear overexpression is related to worse outcome. ${ }^{58}$ Presently, molecular-targeted therapeutic drugs against FGFR2 and c-MET do not appear to be specific for nuclear targets. Our findings may provide evidence for improving the therapeutic effect of c-MET and FGFR2 antibodies.

In the survival analysis, c-MET and FGFR2 overexpression was significantly associated with OS $(P=0.041$ and 0.031 , respectively), which is in line with previous studies. ${ }^{48,51,53,59}$ However, the differences disappeared in multivariate analysis. Contradictory results have been reported on the prognostic value of HER2: one study demonstrated that HER2 was significantly associated with survival, ${ }^{9}$ but another reported opposite results. ${ }^{60}$ In our study, HER 2 overexpression had prognostic significance in both univariate and multivariate analysis $(P<0.05)$.

In the survival analysis, c-MET and FGFR2 overexpression was significantly associated with OS $(P=0.018$ and 0.004 , respectively), which is in line with previous studies. ${ }^{48,51,53,59}$ However, the differences disappeared in multivariate analysis. Contradictory results have been reported on the prognostic value of HER2: one study demonstrated that HER2 was significantly associated with survival, ${ }^{9}$ but another reported opposite results. ${ }^{60}$ In our study, HER2 overexpression had prognostic significance in both univariate and multivariate analysis.

In the present study, $60 \%$ of patients $(85 / 143)$ expressed at least one of the three RTKs, and $29.3 \%$ of patients (42/143) were simultaneously multiple-positive in the same sample, which is in line with recent studies. Recently, Nagatsuma et al ${ }^{48}$ reported the expression patterns of four RTKs by IHC in a large Japanese cohort. They found that $63.1 \%$ of patients with resected GC expressed at least one of four RTKs. Moreover, $22.7 \%$ of cases expressed multiple RTKs simultaneously in the same sample. ${ }^{48} \mathrm{~A}$ study detailing a molecular genomic map of GC demonstrated that, collectively, $37 \%$ of cases exhibit at least one RTK amplification event. ${ }^{61}$ Guo et al explored the expression of four RTKs in a GC cohort and reported that $\sim 90 \%$ of GC tissue showed the upregulation of at least one of four proteins, including c-MET and FGFR2, while all four proteins were overexpressed in $20 \%$ of the tissues. ${ }^{62}$ The coexpression profile of RTKs is of great value for the multitargeting therapy and combination of target drugs in GC.

Based on clinical experience, even within HER2-positive patients with GC, 50\% exhibited primary resistance, and the majority of the initially responding patients developed secondary resistance within a year. Given the common downstream pathway, the alternative activation of RTKs and the crosstalk between the signaling pathways have been considered a significant mechanism contributing to resistance to inhibitors targeting epidermal growth factor receptor (EGFR) family members as well as HER2 in GC. ${ }^{63,64}$ In the present study, the rate of HER $2 / \mathrm{c}-\mathrm{MET}$ coexpression was $7 \%$, that of HER2/FGFR2 was 5.6\%, and that of triple-positive GC was $3.5 \%$. Furthermore, HER2+/c-MET+, HER2+/FGFR2+, and triple-positive status was significantly associated with poor clinical outcome when the cohort was subdivided by coexpression status. Recently, Fuse et al analyzed Japanese patients with GC and characterized the rate of HER2 and c-MET coexpression as $8 \%$, which is in line with our report. ${ }^{65}$ Shattuck et al reported that c-MET signal activation was responsible for trastuzumab resistance in breast cancer. ${ }^{66}$ Khoury et al found that hepatocyte growth factor/c-MET converted HER2-positive breast epithelial morphogenesis to cell invasion as a consequence of cooperation downstream of these signals. ${ }^{67}$ Similarly, Corso et al uncovered poor response to c-MET inhibitors in patients with c-MET overexpression and with HER family member activation. ${ }^{68} \mathrm{Chen}$ et al demonstrated that c-MET activation led to resistance to lapatinib (a dual tyrosine kinase inhibitor: anti-EGFR and anti-HER2) in HER2-amplified GC. ${ }^{63}$ Considering the crosstalk and coexpression between the signaling pathways, it is speculated that c-MET overexpression could potentially be a contributing factor to the low response rates of antiHER2-targeted therapy observed in the clinic. Therefore, combined-targeted therapy of HER2 and c-MET is likely to be a promising approach for overcoming drug resistance and improving the survival of patients with GC.

In conclusion, there is a significant association between RTK co-overexpression, poor clinical outcome, and therapeutic drug resistance. Better understanding of RTK expression status in $\mathrm{GC}$ would allow for patient-tailored 
therapy based on biomarker-assisted population selection. Our study may provide more evidence for further GC multitargeting therapy.

\section{Acknowledgments}

We thank Doctor Zhiwei Chang who provided technical help in this study. We thank Elixigen Corporation (Huntington Beach, California, USA) for helping in proofreading and editing the English of final manuscript.

\section{Disclosure}

The authors report no conflicts of interest in this work.

\section{References}

1. Jemal A, Bray F, Center MM, Ferlay J, Ward E, Forman D. Global cancer statistics. CA Cancer J Clin. 2011;61:69-90.

2. Ferlay J, Shin HR, Bray F, Forman D, Mathers C, Parkin DM. Estimates of worldwide burden of cancer in 2008: GLOBOCAN 2008. Int J Cancer. 2010;127:2893-2917.

3. Brenner H, Rothenbacher D, Arndt V. Epidemiology of stomach cancer. Methods Mol Biol. 2009;472:467-477.

4. Buzzoni R, Bajetta E, Di Bartolomeo M, et al. Pathological features as predictors of recurrence after radical resection of gastric cancer. $\mathrm{Br}$ J Surg. 2006;93:205-209.

5. Power DG, Kelsen DP, Shah MA. Advanced gastric cancer - slow but steady progress. Cancer Treat Rev. 2010;36:384-392.

6. Scartozzi M, Galizia E, Verdecchia L, et al. Chemotherapy for advanced gastric cancer: across the years for a standard of care. Expert Opin Pharmacother. 2007;8:797-808.

7. Bang YJ, Van Cutsem E, Feyereislova A, et al. Trastuzumab in combination with chemotherapy versus chemotherapy alone for treatment of HER2-positive advanced gastric or gastro-oesophageal junction cancer (ToGA): a phase 3, open-label, randomised controlled trial. Lancet. 2010;376:687-697.

8. Hofmann M, Stoss O, Shi D, et al. Assessment of a HER2 scoring system for gastric cancer: results from a validation study. Histopathology. 2008;52:797-805.

9. Tanner M, Hollmen M, Junttila TT, et al. Amplification of HER-2 in gastric carcinoma: association with Topoisomerase II alpha gene amplification, intestinal type, poor prognosis and sensitivity to trastuzumab. Ann Oncol. 2005;16:273-278.

10. Gravalos C, Jimeno A. HER2 in gastric cancer: a new prognostic factor and a novel therapeutic target. Ann Oncol. 2008;19:1523-1529.

11. Bussolino F, Di Renzo MF, Ziche M, et al. Hepatocyte growth factor is a potent angiogenic factor which stimulates endothelial cell motility and growth. J Cell Biol. 1992;119:629-641.

12. Zhang YW, Su Y, Volpert OV, Vande Woude GF. Hepatocyte growth factor/scatter factor mediates angiogenesis through positive VEGF and negative thrombospondin 1 regulation. Proc Natl Acad Sci U SA. 2003;100:12718-12723.

13. Jeffers M, Fiscella M, Webb CP, Anver M, Koochekpour S, Vande Woude GF. The mutationally activated Met receptor mediates motility and metastasis. Proc Natl Acad Sci U S A. 1998;95:14417-14422.

14. Turner N, Grose R. Fibroblast growth factor signalling: from development to cancer. Nat Rev Cancer. 2010;10:116-129.

15. Kunii K, Davis L, Gorenstein J, et al. FGFR2-amplified gastric cancer cell lines require FGFR2 and Erbb3 signaling for growth and survival. Cancer Res. 2008;68:2340-2348.

16. Takeda M, Arao T, Yokote H, et al. AZD2171 shows potent antitumor activity against gastric cancer over-expressing fibroblast growth factor receptor 2/keratinocyte growth factor receptor. Clin Cancer Res. 2007; 13:3051-3057.
17. Brand-Saberi B, Muller TS, Wilting J, Christ B, Birchmeier C. Scatter factor/hepatocyte growth factor (SF/HGF) induces emigration of myogenic cells at interlimb level in vivo. Dev Biol. 1996;179: 303-308.

18. Peschard P, Park M. From Tpr-Met to Met, tumorigenesis and tubes. Oncogene. 2007;26:1276-1285.

19. Ponzetto C, Bardelli A, Zhen Z, et al. A multifunctional docking site mediates signaling and transformation by the hepatocyte growth factor/ scatter factor receptor family. Cell. 1994;77:261-271.

20. Nguyen L, Holgado-Madruga M, Maroun C, et al. Association of the multisubstrate docking protein Gab1 with the hepatocyte growth factor receptor requires a functional Grb2 binding site involving tyrosine 1356. J Biol Chem. 1997;272:20811-20819.

21. Mughal A, Aslam HM, Khan AM, Saleem S, Umah R, Saleem M. Bcr-Abl tyrosine kinase inhibitors - current status. Infect Agent Cancer. 2013;8:23.

22. Peters S, Adjei AA. MET: a promising anticancer therapeutic target. Nat Rev Clin Oncol. 2012;9:314-326.

23. Yamamoto N, Mammadova G, Song RX, Fukami Y, Sato K. Tyrosine phosphorylation of $\mathrm{p} 145 \mathrm{met}$ mediated by EGFR and $\mathrm{Src}$ is required for serum-independent survival of human bladder carcinoma cells. J Cell Sci. 2006;119:4623-4633.

24. Catenacci DV, Henderson L, Xiao SY, et al. Durable complete response of metastatic gastric cancer with anti-Met therapy followed by resistance at recurrence. Cancer Dis. 2011;1:573-579.

25. Beenken A, Mohammadi M. The FGF family: biology, pathophysiology and therapy. Nat Rev Drug Discov. 2009;8:235-253.

26. Courjal F, Cuny M, Simony-Lafontaine J, et al. Mapping of DNA amplifications at 15 chromosomal localizations in 1875 breast tumors: definition of phenotypic groups. Cancer Res. 1997;57:4360-4367.

27. Jang JH, Shin KH, Park JG. Mutations in fibroblast growth factor receptor 2 and fibroblast growth factor receptor 3 genes associated with human gastric and colorectal cancers. Cancer Res. 2001;61:3541-3543.

28. Dutt A, Salvesen HB, Chen TH, et al. Drug-sensitive FGFR2 mutations in endometrial carcinoma. Proc Natl Acad Sci U S A. 2008;105: 8713-8717.

29. Hattori $\mathrm{Y}$, Itoh $\mathrm{H}$, Uchino $\mathrm{S}$, et al. Immunohistochemical detection of K-sam protein in stomach cancer. Clin Cancer Res. 1996;2: $1373-1381$.

30. Ueda T, Sasaki H, Kuwahara Y, et al. Deletion of the carboxyl-terminal exons of K-sam/FGFR2 by short homology-mediated recombination, generating preferential expression of specific messenger RNAs. Cancer Res. 1999;59:6080-6086.

31. Cappellen D, De Oliveira C, Ricol D, et al. Frequent activating mutations of FGFR3 in human bladder and cervix carcinomas. Nat Genet. 1999;23:18-20.

32. Guagnano V, Kauffmann A, Wohrle S, et al. FGFR genetic alterations predict for sensitivity to NVP-BGJ398, a selective pan-FGFR inhibitor. Cancer Discov. 2012;2:1118-1133.

33. Zhang J, Zhang L, Su X, et al. Translating the therapeutic potential of AZD4547 in FGFR1-amplified non-small cell lung cancer through the use of patient-derived tumor xenograft models. Clin Cancer Res. 2012;18:6658-6667.

34. Gavine PR, Mooney L, Kilgour E, et al. AZD4547: an orally bioavailable, potent, and selective inhibitor of the fibroblast growth factor receptor tyrosine kinase family. Cancer Res. 2012;72:2045-2056.

35. Zhao WM, Wang L, Park H, et al. Monoclonal antibodies to fibroblast growth factor receptor 2 effectively inhibit growth of gastric tumor xenografts. Clin Cancer Res. 2010;16:5750-5758.

36. Kim JW, Im SA, Kim M, et al. The prognostic significance of HER2 positivity for advanced gastric cancer patients undergoing first-line modified FOLFOX-6 regimen. Anticancer Res. 2012;32:1547-1553.

37. Lee HE, Kim MA, Lee HS, et al. MET in gastric carcinomas: comparison between protein expression and gene copy number and impact on clinical outcome. Br J Cancer. 2012;107:325-333.

38. Matsumoto K, Arao T, Hamaguchi T, et al. FGFR2 gene amplification and clinicopathological features in gastric cancer. Br J Cancer. 2012; 106:727-732. 
39. Lauren $P$. The two histological main types of gastric carcinoma: diffuse and so-called intestinal-type carcinoma. an attempt at a histo-clinical classification. Acta Pathol Microbiol Scand. 1965;64:31-49.

40. Mullaney PJ, Wadley MS, Hyde C, et al. Appraisal of compliance with the UICC/AJCC staging system in the staging of gastric cancer. Union Internacional Contra la Cancrum/American Joint Committee on Cancer. Br J Surg. 2002;89:1405-1408.

41. Spigel DR, Ervin TJ, Ramlau RA, et al. Randomized phase II trial of Onartuzumab in combination with erlotinib in patients with advanced non-small-cell lung cancer. J Clin Oncol. 2013;31:4105-4114.

42. Konecny GE, Finkler N, Garcia AA, et al. Second-line dovitinib (TKI258) in patients with FGFR2-mutated or FGFR2-non-mutated advanced or metastatic endometrial cancer: a non-randomised, openlabel, two-group, two-stage, phase 2 study. Lancet Oncol. 2015;16: 686-694.

43. Chua TC, Merrett ND. Clinicopathologic factors associated with HER2-positive gastric cancer and its impact on survival outcomes - a systematic review. Int J Cancer. 2012;130:2845-2856.

44. Nakajima M, Sawada H, Yamada Y, et al. The prognostic significance of amplification and overexpression of c-met and c-erb B-2 in human gastric carcinomas. Cancer. 1999;85:1894-1902.

45. Yu S, Yu Y, Zhao N, Cui J, Li W, Liu T. C-Met as a prognostic marker in gastric cancer: a systematic review and meta-analysis. PLoS One 2013;8:e79137.

46. Acunzo M, Romano G, Palmieri D, et al. Cross-talk between MET and EGFR in non-small cell lung cancer involves miR-27a and Sprouty2. Proc Natl Acad Sci U S A. 2013;110:8573-8578.

47. Park YS, Na YS, Ryu MH, et al. FGFR2 assessment in gastric cancer using quantitative real-time polymerase chain reaction, fluorescent in situ hybridization, and immunohistochemistry. Am J Clin Pathol. 2015;143:865-872.

48. Nagatsuma AK, Aizawa M, Kuwata T, et al. Expression profiles of HER2, EGFR, MET and FGFR2 in a large cohort of patients with gastric adenocarcinoma. Gastric Cancer. 2015;18:227-238.

49. Shan L, Ying J, Lu N. HER2 expression and relevant clinicopathological features in gastric and gastroesophageal junction adenocarcinoma in a Chinese population. Diagn Pathol. 2013;8:76.

50. Sheng WQ, Huang D, Ying JM, et al. HER2 status in gastric cancers: a retrospective analysis from four Chinese representative clinical centers and assessment of its prognostic significance. Ann Oncol. 2013; 24:2360-2364.

51. Ha SY, Lee J, Kang SY, et al. MET overexpression assessed by new interpretation method predicts gene amplification and poor survival in advanced gastric carcinomas. Modern Pathol. 2013;26:1632-1641.

52. Sotoudeh K, Hashemi F, Madjd Z, Sadeghipour A, Molanaei S, Kalantary E. The clinicopathologic association of c-MET overexpression in Iranian gastric carcinomas; an immunohistochemical study of tissue microarrays. Diagn Pathol. 2012;7:57.

53. Amemiya H, Kono K, Itakura J, et al. c-Met expression in gastric cancer with liver metastasis. Oncology. 2002;63:286-296.
54. Terashima M, Kitada K, Ochiai A, et al. Impact of expression of human epidermal growth factor receptors EGFR and ERBB2 on survival in stage II/III gastric cancer. Clin Cancer Res. 2012;18:5992-6000.

55. Okines AF, Thompson LC, Cunningham D, et al. Effect of HER2 on prognosis and benefit from peri-operative chemotherapy in early oesophago-gastric adenocarcinoma in the MAGIC trial. Ann Oncol. 2013; 24:1253-1261

56. Shitara K, Yatabe Y, Matsuo K, et al. Prognosis of patients with advanced gastric cancer by HER2 status and trastuzumab treatment. Gastric Cancer. 2013;16:261-267.

57. Wiedlocha A, Falnes PO, Madshus IH, Sandvig K, Olsnes S. Dual mode of signal transduction by externally added acidic fibroblast growth factor. Cell. 1994;76:1039-1051.

58. Behrens C, Lin HY, Lee JJ, et al. Immunohistochemical expression of basic fibroblast growth factor and fibroblast growth factor receptors 1 and 2 in the pathogenesis of lung cancer. Clin Cancer Res. 2008;14:6014-6022.

59. Betts G, Valentine H, Pritchard S, et al. FGFR2, HER2 and cMet in gastric adenocarcinoma: detection, prognostic significance and assessment of downstream pathway activation. Virchows Arch. 2014;464: $145-156$.

60. Grabsch H, Sivakumar S, Gray S, Gabbert HE, Muller W. HER2 expression in gastric cancer: rare, heterogeneous and of no prognostic value - conclusions from 924 cases of two independent series. Cell Oncol. 2010;32:57-65.

61. Deng N, Goh LK, Wang H, et al. A comprehensive survey of genomic alterations in gastric cancer reveals systematic patterns of molecular exclusivity and co-occurrence among distinct therapeutic targets. Gut. 2012;61:673-684

62. Guo T, Fan L, Ng WH, et al. Multidimensional identification of tissue biomarkers of gastric cancer. J Proteome Res. 2012;11:3405-3413.

63. Chen CT, Kim H, Liska D, Gao S, Christensen JG, Weiser MR. MET activation mediates resistance to lapatinib inhibition of HER2-amplified gastric cancer cells. Mol Cancer Ther. 2012;11:660-669.

64. Kneissl J, Keller S, Lorber T, et al. Association of amphiregulin with the cetuximab sensitivity of gastric cancer cell lines. Int J Oncol. 2012; 41:733-744.

65. Fuse N, Kuboki Y, Kuwata T, et al. Prognostic impact of HER2, EGFR, and c-MET status on overall survival of advanced gastric cancer patients. Gastric Cancer. 2016;19:183-191.

66. Shattuck DL, Miller JK, Carraway KL 3rd, Sweeney C. Met receptor contributes to trastuzumab resistance of Her2-overexpressing breast cancer cells. Cancer Res. 2008;68:1471-1477.

67. Khoury H, Naujokas MA, Zuo D, et al. HGF converts ErbB2/Neu epithelial morphogenesis to cell invasion. Mol Biol Cell. 2005;16:550-561.

68. Corso S, Ghiso E, Cepero V, et al. Activation of HER family members in gastric carcinoma cells mediates resistance to MET inhibition. Mol Cancer. 2010;9:121.
OncoTargets and Therapy

\section{Publish your work in this journal}

OncoTargets and Therapy is an international, peer-reviewed, open access journal focusing on the pathological basis of all cancers, potential targets for therapy and treatment protocols employed to improve the management of cancer patients. The journal also focuses on the impact of management programs and new therapeutic agents and protocols on

\section{Dovepress}

patient perspectives such as quality of life, adherence and satisfaction. The manuscript management system is completely online and includes a very quick and fair peer-review system, which is all easy to use. Visit http://www.dovepress.com/testimonials.php to read real quotes from published authors. 